\title{
Ventral Striatal Blood Flow is Altered by Acute Nicotine but Not Withdrawal from Nicotine
}

\author{
Jody Tanabe*,I,2, Thomas Crowley², Kent Hutchison ${ }^{3}$, David Miller', Glyn Johnson ${ }^{4}$, Yiping P Du ${ }^{1,2}$, \\ Gary Zerbe Z,5 $^{2,5}$ Robert Freedman ${ }^{2}$ \\ 'Department of Radiology, University of Colorado at Denver Health Sciences, Denver, CO, USA; ${ }^{2}$ Department of Psychiatry, University of \\ Colorado at Denver Health Sciences, Denver, CO, USA; ${ }^{3}$ Department of Psychology, University of Colorado at Boulder, Denver, CO, USA; \\ ${ }^{4}$ Department of Radiology, New York University Medical Center, New York, NY, USA; ${ }^{5}$ Department of Biometrics, University of Colorado \\ at Denver Health Sciences, Denver, CO, USA
}

\begin{abstract}
Neural mechanisms underlying the reinforcing effects of nicotine and other drugs have been widely studied and are known to involve the ventral striatum, which is part of the mesocorticolimbic dopamine system. In contrast, mechanisms of nicotine withdrawal have received less attention although subjective withdrawal likely contributes to the difficulty of quitting. The goal of this study was to determine if nicotine withdrawal was associated with alterations of cerebral blood flow (CBF) in ventral striatum. Twelve smokers, moderately dependent on nicotine, underwent MR dynamic susceptibility contrast (DSC) imaging at baseline, after overnight withdrawal from nicotine, and after nicotine replacement. DSC images were used to calculate CBF in three regions of interest: ventral striatum, thalamus, and medial frontal cortex. Subjective withdrawal symptoms were measured at each time point. In spite of significant subjective withdrawal symptoms, there was no main effect of withdrawal on CBF in the three regions. However, there was a significant correlation between the increase in withdrawal symptoms and a reduction in thalamic CBF. In contrast to withdrawal, nicotine replacement significantly increased CBF in ventral striatum. Our findings are consistent with the known role of ventral striatum in drug reward. The lack of a main effect on withdrawal, but correlation of thalamic blood flow with withdrawal symptoms suggests that more complex mechanisms mediate the subjective features of the withdrawal state.
\end{abstract}

Neuropsychopharmacology (2008) 33, 627-633; doi:I0.1038/sj.npp. I30I428; published online 25 April 2007

Keywords: nicotine; subjective withdrawal; cerebral blood flow; ventral striatum

\section{INTRODUCTION}

The majority of first time and relapsed smokers have a high interest in quitting (Fu et al, 2006). While smoking rates dramatically decreased in the 1960 s and continue to decrease among US adults, the rate of cessation has slowed recently and the prevalence of smoking remains high, especially in certain segments of the population (CDC, 2005). Physical dependence on nicotine and the desire to avoid withdrawal symptoms likely contribute to the difficulty in quitting. Similar to other drugs, withdrawal from nicotine may be objective or subjective. Animal studies provide insights into mechanisms of objective withdrawal, but certain subjective changes can only be studied in humans. Cellular adaptations due to chronic nicotine likely mediate a withdrawal syndrome, but the

*Correspondence: Dr J Tanabe, Department of Radiology and Psychiatry, University of Colorado Health Sciences Center, Box A034, 4200 East 9th Avenue, Denver, CO 80262, USA, Tel: + I 303372 6539, Fax: + I 303372 6148,

E-mail: jody.tanabe@uchsc.edu

Received 29 October 2006; revised 8 March 2007; accepted 20 March 2007 neural systems implicated are wide-ranging and the subject of speculation. For example, there is evidence that dopaminergic, serotonergic, opioid, cholinergic, and corticotrophin neurotransmitter systems may all play a role in nicotine withdrawal (Watkins et al, 2000). Animal studies suggest that the mesolimbic system known to be involved in acute reinforcement may also mediate nicotine withdrawal (Watkins et al, 2000; Markou et al, 1998; EppingJordan et al, 1998). For example, reductions in extracellular dopamine in nucleus accumbens, but not in prefrontal cortex, has been observed following pharmacologic nicotine withdrawal in rats (Watkins et al, 2000; Hildebrand et al, 1998).

Previous neuroimaging studies investigating the effects of nicotine in smokers have mainly used positron emission tomography (PET) methods. Domino et al (2000c, 2000a) found that compared to pepper spray, nicotine spray increased glucose metabolism in inferior frontal lobe, posterior cingulate gyrus, and thalamus and increased cerebral blood flow (CBF) in thalamus, pons, and Brodmann area (BA)17. Zubieta et al (2001) measured the effect of intranasal nicotine in 10 regions of interest (ROI). Nicotine was associated with increases in regional CBF 
( $\mathrm{rCBF}$ ) in thalamus, a structure with high nicotinic receptor density, and decreases in $\mathrm{rCBF}$ in anterior temporal lobe and amygdala. After smoking a cigarette containing nicotine, smokers showed an increase in $\mathrm{CBF}$ in visual cortex and cerebellum, two structures also rich in nicotinic cholinergic receptors, and a reduction in ventral striatum (Zubieta et al, 2005). Intravenous nicotine increased CBF in parieto-occipital cortex and decreased CBF in anterior cingulate and cerebellum in smokers (Ghatan et al, 1998). In an attempt to elucidate neural systems rather than single brain regions activated by nicotine, Rose et al (2003a) used a principal component analysis to group multiple regions contributing to either striatal, reticular, or frontal factors. They observed a dose-related CBF response in the right reticular system factor that correlated with craving.

While most of these studies required abstinence from cigarettes before baseline imaging, none specifically hypothesized changes in CBF associated with acute abstinence or withdrawal. The spatial resolution of PET data is lower than that of MRI both in the acquired data and as a result of smoothing. Potential artifacts due to partial volume averaging associated with PET imaging may not be trivial when considering the relatively small volume of structures of interest such as ventral striatum. A functional MRI (fMRI) study of the effects of intravenous nicotine demonstrated increased activity in the cingulate, several divisions of the frontal lobe, occipital lobe, nucleus accumbens, amygdala, and limbic thalamic nuclei in a setting of ad lib smoking. Activity was defined as those voxels fulfilling six criteria based on a pharmacokinetic waveform of nicotine (Stein et al, 1998). Compared with this waveform model, dynamic susceptibility contrast (DSC) MR imaging is a more direct physiological measurement of cerebral hemodynamics that maintains the higher spatial resolution of MR imaging.

We used DSC-MR imaging to determine if acute withdrawal from and administration of nicotine would alter CBF in the ventral striatum, defined anatomically using a ROI approach. Two control ROIs were the thalamus, given its known high density of nicotinic cholinergic receptors, and frontal gray matter.

\section{METHODS}

\section{Subjects}

Seven female and five male smokers were recruited through flyers and newspaper advertisements. The mean $( \pm S D)$ age was $27.7(9.2)$ years. Subjects were included if they had no current or past history of serious medical, neurological, or psychiatric illness, including non-nicotine substance abuse or dependence. Written informed consent was obtained from all subjects. All procedures were approved by the Colorado Multiple Institutional Review Board. Subjects had smoked for an average of 8.2 (7.1) years: $75 \%$ of subjects smoked 21-30 cigarettes per day whereas $25 \%$ smoked $11-20$ cigarettes per day. The mean score on the Fagerstrom Test for Nicotine Dependence (FTND) questionnaire was 5.42 (1.4), indicating moderate nicotine dependence. Subjects arrived at the MR scanner at 1600 hours after smoking their usual amount that day. Baseline measurements were completed by 1800 hours and included breath carbon monoxide (CO) levels (Vitalograph USA, Lenexa, KS), vital signs, behavioral questions, and MR scans. Subjects were then asked to abstain from smoking overnight and return at 0800 hours the following day. Abstinence was confirmed by self-report, CO levels $\leqslant 10$ p.p.m., and a reduction in CO levels from baseline. Thus, subjects were abstinent between 16 and $18 \mathrm{~h}$. On the second day, behavioral questions and MR scans were obtained during acute withdrawal and after receiving nicotine gum (described in detail below).

An 18 gauge IV catheter was inserted into an antecubital vein in each subject before entering the MR scanner. The catheter was connected to an MR compatible power injector (MedRad, Indianola, PA) and the vein was kept patent by saline drip. The catheter was removed at the end of the scan on day 1 and a new catheter was inserted in the same vein on day 2.

\section{Behavioral Questions}

Questionnaires were given at the 1600 hour session, after overnight abstinence and after nicotine gum as follows:

\section{Baseline (1600 hours)}

Smoking history questionnaire collected information on frequency and quantity of current nicotine use, history of nicotine use, and previous quit attempts. Nicotine Dependence Syndrome Scale is a measure of nicotine dependence that may complement the FTND (Colby et al, 2000). Minnesota Nicotine Withdrawal Scale is a multiple item scale for tobacco withdrawal (Hughes and Hatsukami, 1986). The scale included seven DSM-IV items and excluded craving and somatic items (Hughes and Hatsukami, 1998). Smoking Complaint Scale (SCS), a self-report questionnaire used to assess tobacco withdrawal, consists of 20 items scored on a seven-point Likert scale (Schneider et al, 1984).

\section{Withdrawal (After Overnight Abstinence)}

On day 2, after overnight abstinence and immediately before MR scanning, subjects completed the Minnesota Nicotine Withdrawal Scale and SCS.

\section{Post-nicotine Gum}

After completion of MR scanning, subjects completed the Minnesota Nicotine Withdrawal Scale and SCS, approximately $20 \mathrm{~min}$ after having received nicotine gum.

\section{MR Acquisition}

Images were acquired with a $3 \mathrm{~T}$ GE whole body MR system (General Electric, Milwaukee, WI) using an eight-channel head coil. At the time of study, our scanner did not have the capability of performing parallel imaging. Head position was maintained by fitting a head-conforming vacuum cushion (Vac-Fix, Par Scientific A/S, Denmark) centered at the base of the subject's occiput. The conformation of the pillow was maintained for all sessions to ensure that head angle relative to the neck remained as similar as possible 
across sessions. Two conventional images were acquired: (i) high-resolution 3D T1-weighted anatomical scan for image registration and anatomical delineation (Inversion prepared fast SPGR, TR $9.1 \mathrm{~ms} / \mathrm{TI} 500 \mathrm{~ms} / \mathrm{TE} 2 \mathrm{~ms} / \mathrm{FA} 10$, $0.86 \times 0.86 \times 1.7 \mathrm{~mm}^{3}$ ), (ii) axial 2 -D spin-echo T1-weighted images in the same plane and of the same slice thickness and gap as the CBF images were used to optimize co-registration between the anatomical images and $\mathrm{CBF}$ maps.

\section{CBF Using First-Pass DSC Imaging}

Automated high-order shimming was performed before each CBF acquisition. MR images were acquired with gradient-echo echo-planar imaging during the first pass of a standard bolus $(0.1 \mathrm{mmol} / \mathrm{kg})$ of gadodiamide (Omniscan, Amersham Health). Imaging parameters were TR 1500/TE 22.5/FA 80, 18-20 axial slices centered on the inferior frontal lobe, in-plane resolution $1.9 \times 1.9 \mathrm{~mm}^{2} \quad(\mathrm{FOV}$ $240 \mathrm{~mm}^{2}$, matrix $\left.128 \times 128\right)$, 3-mm-thick slices with $1 \mathrm{~mm}$ gap, readout bandwidth $62 \mathrm{kHz}$. Contrast was injected at the 10th acquisition at a rate of $5 \mathrm{ml} / \mathrm{s}$ followed by a $20 \mathrm{ml}$ bolus of saline solution at $5 \mathrm{ml} / \mathrm{s}$. On the second day, three doses of contrast were administered: after overnight abstinence ('withdrawal 1'), $10 \mathrm{~min}$ later ('withdrawal 2'), and after chewing nicotine gum ('post-nicotine'). Measurements at the time of 'withdrawal 2' were obtained to minimize the effects of residual contrast due to sequential bolus administration (Levin et al, 1998) against which we compared the 'post-nicotine' measurements.

\section{Nicotine Gum}

Nicotine was administered as nicotine polacrilex (Nicorette) gum $6 \mathrm{mg}$ (three $2 \mathrm{mg}$ pieces pressed together). Subjects were taken out of the MR scanner, sat up, and remained on the scanner table. They chewed the gum for $10 \mathrm{~min}$ and were asked not to swallow saliva but, instead, spit the saliva into a cup. Using this procedure, venous nicotine levels are expected to peak about $5 \mathrm{~min}$ after chewing begins (Harris et al, 2004) which is approximately the time the 'postnicotine' MR scan was acquired. Blood pressure and heart rate were recorded with MR compatible monitors. After the 10 -min gum chewing session, subjects were repositioned in the magnet. All subjects tolerated $6 \mathrm{mg}$ of nicotine gum without adverse reaction.

\section{Image Analysis}

Calculation of CBF maps. Perfusion analysis was performed with programs developed in-house using the IDL programming languages based on the method of Rempp et al (1994a) and Ostergaard et al (1996). Contrast agent concentration, $C$, is first found using the simple relationship (Rosen et al, 1991)

$$
C \propto-\ln \left(\frac{S}{S_{0}}\right)
$$

where $S$ is the signal intensity and $S_{0}$ is the pre-bolus signal intensity. This equation assumes that $\mathrm{T} 1$ shortening effects are negligible which is true in practice since we use a relatively low flip angle to minimize saturation.
Mean transit time (MTT) and cerebral blood volume $(\mathrm{CBV})$ are then calculated using the following equations:

$$
\begin{aligned}
& \text { MTT }=\frac{\int C \mathrm{~d} t}{C_{\max }} \\
& \mathrm{CBV}=\frac{\int C \mathrm{~d} t}{\int \mathrm{AIFdt}}
\end{aligned}
$$

Finally, CBF is calculated from the simple relationship

$$
\mathrm{CBF}=\frac{\mathrm{CBV}}{\mathrm{MTT}}
$$

where $C$ is the tissue concentration following an ideal, instantaneous bolus and $C_{\max }$ is the maximum value of $C$. The bolus is not instantaneous, of course, but an approximation to the idealized response can be found by deconvolving the measured tissue concentration with the arterial input function (AIF). The AIF was found using an automated method similar to that described by Rempp et al (1994b) and Carroll et al (2003). The minimum signal, corresponding to the bolus peak, is found in each pixel within the scan. The average signal drop and average bolus arrival time are then calculated for all pixels. Pixels where the bolus arrives early and where the signal drop is larger than average are assumed to be within arteries. The AIF is then calculated by averaging the signals from all such pixels.

Region of interest. Three ROIs corresponding to ventral striatum, thalamus, and medial frontal gray matter were manually traced and edited on each subjects' 3D anatomical image using BRAINS2 Software (Magnotta et al, 2002). The final ROI was converted to Analyze format for subsequent analysis using MRICro (Rorden and Brett, 2000). Ventral striatum was defined based on a modification of landmarks described by Mawlawi et al (2001) that showed good correspondence with dopamine D2 receptor distribution. The ventral striatum as defined by Mawlawi included the ventral lateral pallidum as well as the ventral medial striatum (eg nucleus accumbens) and may be more inclusively termed the ventral basal ganglia. Since a discrete anatomical border between lateral pallidum and medial striatum (which includes nucleus accumbens) is difficult to define, we operationally excluded the lateral $1 / 3$ of the ventral basal ganglia specified by the Mawlawi landmarks. An example of a ventral striatum ROI is shown in Figure 1. Medial frontal gray matter was defined by applying a signal intensity threshold to a spherical ROI of approximately $5 \mathrm{~mm}^{3}$ placed in the center of the right posterior medial frontal cortex. We used a conservative intensity threshold to minimize partial volume averaging from adjacent white matter or sulcal CSF. Thalamus was also conservatively defined so as to exclude partial volume averaging from third ventricular CSF. Spheres of approximately $5 \mathrm{~mm}^{3}$ were placed in the medial right and left thalami. All ROIs were visually inspected to ensure that pixels of the ROI were in gray matter and did not include adjacent CSF or white matter.

Image registration. Anatomic localization of the ventral striatum is best performed using the highest spatial resolution possible (eg 3D-T1 gradient echo anatomical). 


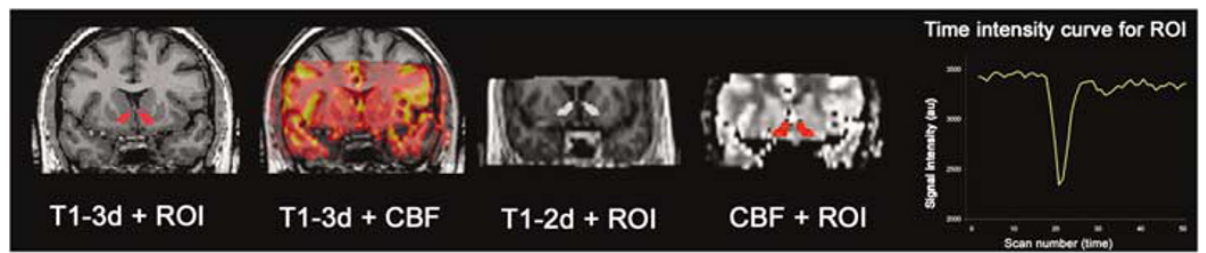

Figure I Example of image registrations between 3D-TI anatomical, ventral striatum ROI, 2D-TI partial head, and CBF partial head. All images were transformed to native anatomical space. The 2D TI data set was co-registered to the 3D TI data set, providing the geometric transform needed to move each CBF map to native anatomical space (see Methods for details). Time-intensity curve for the ROI is shown at far right.

All subsequent images were registered to native anatomical space. Given the small volume of our ROIs, we chose to maximize spatial resolution of the CBF maps at the expense of full-head coverage. To compensate for registration errors related to this partial-head coverage, geometric distortions, and noise, each of the four CBF data sets (baseline, withdrawal1, withdrawal 2, and post-nicotine) was acquired with a 2D T1-weighted partial head. The paired 2D T1 and CBF data sets were acquired at the same slice angle, thickness, and gap. The 2D T1 data set was co-registered to the 3D T1 data set, providing the geometric transform needed to move each CBF map to native anatomical space. Registrations were performed using SPM2 (Statistical Parametric Mapping, nine degrees of freedom: three translations, three rotations, and three scales). An example of the registrations is shown Figure 1.

\section{Statistical Analysis}

Statistical analyses were performed using SPSS. Dependent variables (CBF and behavioral measurements) met assumptions of normality. The data were analyzed by repeated measures analysis of variance (rmANOVA) with an unstructured covariance matrix (Cole and Grizzle, 1966) with emphasis on a priori comparisons of baseline to withdrawal 1 , withdrawal 1 to withdrawal 2 , and withdrawal 2 to postnicotine. We did not correct for multiple comparisons for the three regions because we had an a priori hypothesis that ventral striatal $\mathrm{CBF}$ would differ under conditions of abstinence and nicotine replacement and CBF in the other regions would not. Repeated measures ANOVA was used to analyze heart rate and blood pressure following nicotine gum. For behavioral measures comparisons were baseline to withdrawal and withdrawal to post-nicotine. Correlations between behavioral measures and CBF were made using nonparametric Spearman correlations.

\section{RESULTS}

\section{Gender Differences}

No significant gender differences were found in age (women: mean $=25.4$ years, $\mathrm{SD}=8.2$; men: mean $=30.8$ years, $\mathrm{SD}=10.4$ ), number of years smoked (women: mean $=8.1$ years, $S D=5.7$; men: mean $=12.4$ years, $\mathrm{SD}=8.8$ ), FTND (women: mean $=4.9, \quad \mathrm{SD}=1.3$; men: mean $=6.2, \mathrm{SD}=1.1)$, and number of cigarettes smoked per day (women: mean $=20.0, \mathrm{SD}=5.2$; men: mean $=25.0$, $\mathrm{SD}=3.5$ ). There were no main effects of gender on CBF responses; therefore, the data for men and women were combined for all analyses.

\section{Overnight Abstinence}

All subjects were able to abstain from smoking for at least $16 \mathrm{~h}$ overnight, as assessed by self-report and reduction in exhaled CO. Mean CO was 23.0 p.p.m. $(\mathrm{SD}=6.5$, range $16-37)$ at baseline and $6.3(\mathrm{SD}=1.6$, range $4-10)$ after abstinence.

\section{Behavioral Measures}

All subjects completed the Minnesota Withdrawal Questionnaire. Subjects (10 of 12) completed the SCS. There was a significant increase in withdrawal following overnight abstinence as measured on the MWS and SCS. Pairwise comparisons of baseline-withdrawal and withdrawal-nicotine were significant for all measures (paired difference mean \pm SD): $S C S_{\text {baseline }}-\mathrm{SCS}_{\text {withdrawal }}:-23.9 \pm 18.2(95 \% \mathrm{CI}$ : -36.9 to -10.2$), p=0.002 ; \mathrm{SCS}_{\text {withdrawal }}-\mathrm{SCS}_{\text {nicotine, }} 15.5 \pm 14.1$ (95\% CI: 5.4-25.6), $p=0.007 ; \mathrm{MWS}_{\text {baseline }}-\mathrm{MWS}_{\text {withdrawal }}$ : $-1.3 \pm 1.2$ ( $95 \%$ CI: -2.1 to -0.5$), p=0.004$; $\mathrm{MWS}_{\text {withdrawal }}-$ MWS $_{\text {nicotine, }} \quad 0.93 \pm 1.0 \quad(95 \% \quad$ CI: $0.3-1.6), \quad p=0.01$, Craving $_{\text {baseline }}-$ Craving $_{\text {withdrawal, }}-1.9 \pm 0.9$ (95\% CI: -2.5 to $-1.3), \quad p<0.001$; Craving withdrawal - Craving $_{\text {nicotine }}, \quad 2.0 \pm 1.0$ (95\% CI 1.34-2.66), $p<0.001$.

\section{Hemodynamic}

Heart rate and mean arterial blood pressure were monitored every minute during $10 \mathrm{~min}$ of nicotine gum chewing. There was a significant main effect of time on heart rate $(\mathrm{F}=3.8$, df $9, p=0.003)$ and mean arterial blood pressure $(\mathrm{F}=3$, df $9, p=0.01)$ that fit linear and quadratic contrasts, respectively (Figure 2 ).

\section{Regional CBF}

There was a small, but significant increase in CBF in the ventral striatum following nicotine gum: $(51.1$ (5.4) vs 56.2 (8.8) $\mathrm{ml} / \mathrm{cm}^{3} / \mathrm{min}, t=2.5$, df $11, p=0.03$, withdrawal $v s$ nicotine, mean $(\mathrm{SD}))$. There was no difference in ventral striatal CBF during withdrawal or between two consecutive withdrawal measurements. There were no significant differences in perfusion in frontal gray matter or thalamus during any planned comparison (Figure 3 ).

\section{Correlation between rCBF and Behavioral Measurements}

Withdrawal. There was a significant correlation between the change in Minnesota withdrawal score and the change in $\mathrm{CBF}$ in thalamus $(\rho=0.8, p=0.001$, Spearman; Figure 4$)$. 

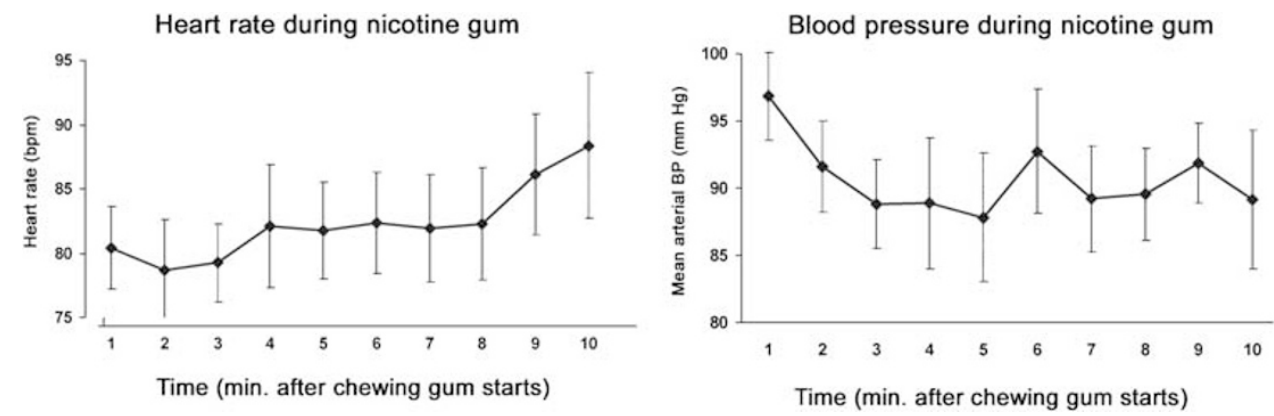

Figure 2 Heart rate and mean arterial blood pressure during nicotine gum chewing. Data are mean and SEM. There was a significant main effect of time on heart rate $(F=3.8, \mathrm{df} 9, p=0.003)$ and mean arterial blood pressure $(F=3, \mathrm{df} 9, p=0.0 \mathrm{I})$ that fit linear and quadratic contrasts, respectively.

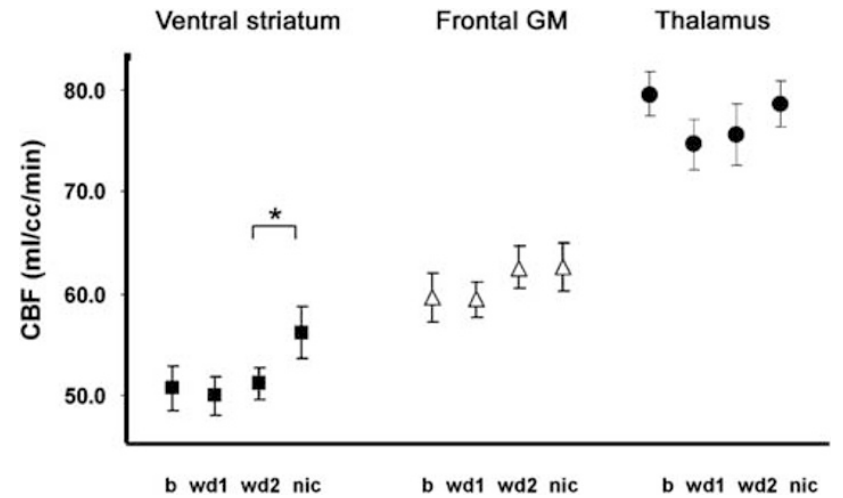

Figure $3 \mathrm{CBF}$ in ventral striatum, frontal gray matter, and thalamus at baseline (b), during withdrawal (wdI), 10 min after wdI (wd2), and after chewing nicotine gum (nic). There was a small, but significant increase in $\mathrm{CBF}$ in the ventral striatum following nicotine gum (mean (SEM)) $(51.1$ (I.5) vs 56.2 (2.5) $\mathrm{ml} / \mathrm{cc} / \mathrm{min}, t=2.3$, df II, * $p=0.03$, wd2 vs nic).

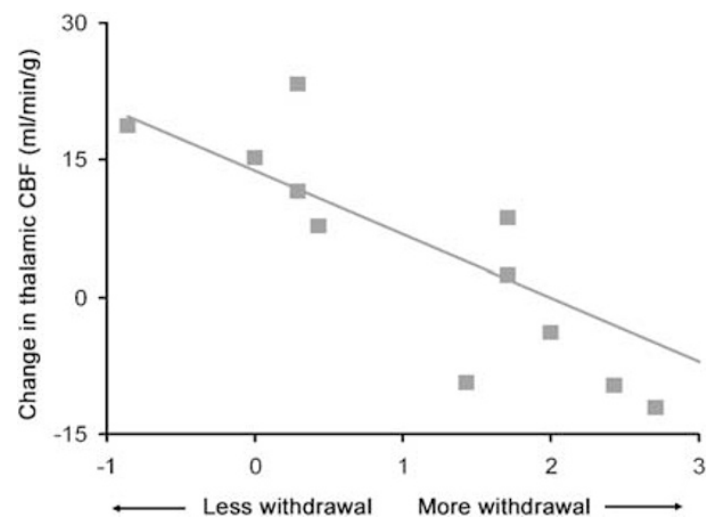

Figure 4 Correlation between change CBF in thalamus and change in Minnesota Withdrawal Score from baseline to withdrawal I. The greater the withdrawal symptoms, the greater the reduction in thalamic CBF.

The greater the withdrawal symptoms, the greater the reduction in thalamic $\mathrm{CBF}$. There were no correlations between changes in SCS and CBF for any region. There were no correlations between $\mathrm{CBF}$ and subjective scores following nicotine.

\section{DISCUSSION}

Subjective symptoms of nicotine withdrawal, as defined in the DSM-IV classification, include irritability, anxiety, restlessness, impatience, depressed mood, and difficulty concentrating. In this study, short-term overnight abstinence elicited significant subjective withdrawal symptoms in moderate smokers, but was not associated with significant changes in CBF in ventral striatum, frontal cortex, or the thalamus. Although there was no main effect of withdrawal on CBF, a significant correlation was observed between changes of blood flow in thalamus and subjective symptoms: the greater the reduction in CBF, the more severe were the subjective symptoms. While we cannot entirely exclude a mechanistic role for the ventral striatum, it may be more complex than can be gleaned from blood flow studies performed following a brief overnight abstinence. In contrast, the administration of nicotine $(6 \mathrm{mg}$ nicotine gum in this study) is a sufficiently potent pharmacological dose to produce a change in ventral striatal blood flow. Our findings are consistent with a known role of ventral striatum mediating acute effects of drug reward.

While the rewarding effects of abused drugs including nicotine clearly involve the mesolimbic dopamine system, the same understanding of brain systems mediating the aversive properties of withdrawal has not been established. There have been many neuroimaging studies of the effects of acute nicotine administration, but none that specifically investigated withdrawal. One difficulty is in defining the onset and duration of withdrawal. Researchers have observed a 'peak' in negative withdrawal symptoms after the first week of abstinence, with a return to baseline by the fourth week. West et al (1989) found this time frame to be consistent across 147 subjects, but there may also be a 'late' withdrawal at approximately 8 weeks (Piasecki et al, 1998). We chose to investigate acute overnight withdrawal to afford consistency across subjects, maximize compliance, optimize image registration in a naturalistic setting, and based on studies showing that blood nicotine levels reach a trough the morning after overnight abstinence, corresponding to the greatest pharmacodynamic and subjective responses associated with the first few cigarettes of the day (Benowitz, 1988). Among smokers, whether the favorite cigarette of the day is the 'first morning' or 'after dinner' depends on the degree of nicotine dependence and pattern of smoking. Infrequent 'chippers' preferred the after-dinner whereas the most nicotine dependent smokers preferred the first morning cigarette (Jarvik et al, 1993). Inconsistent behavioral or physiological results may be related to either the type of smoker or the high variability in the temporal profile of withdrawal. Although nicotine withdrawal can be 
severe, in most cases it is not. It has been suggested that smokers who experience chronic, low-grade withdrawal are more likely to relapse than those whose symptoms abate quickly (Hughes, 2001), underscoring the important role that duration plays in predicting relapse. Few studies have examined physiological correlates of intermediate to longterm withdrawal. Gilbert et al (2004) compared EEG recordings before and after smoking cessation in individuals randomized to quit or not quit. There was more low-frequency EEG activity in the quitters compared to nonquitters that began immediately (day 3 ) and persisted for 31 days after quitting. The cognitive correlate of this lowfrequency activity is a generally reduced arousal state. Since thalamic and reticular nuclei rich in cholinergic activity are involved in general arousal, it is interesting to note that in our study changes in thalamic CBF correlated significantly with changes in subjective withdrawal $(\rho=0.8, p=0.001)$.

Our finding of an increase in CBF in the ventral striatum after nicotine administration is inconsistent with other reports. Previous CBF studies have used $\left[{ }^{15} \mathrm{O}\right] \mathrm{H}_{2} \mathrm{O}-\mathrm{PET}$ to measure $\mathrm{CBF}$ following smoking or nasal spray in overnight abstinent smokers. Zubieta et al (2005) found relative decreases in CBF in nucleus accumbens in nicotinized compared with de-nicotinized cigarettes. Rose et al (2003c) used a principal component analysis to define three factors (frontal, striatal, and reticular systems). In their study, nicotine had no effect on blood flow in the striatal system (defined as caudate, putamen, and posterior cingulate) and reduced flow on the frontal system (ventral striatum, prefrontal cortex, and anterior cingulate). While the grouping of different brain regions to define a 'factor' may uncover important brain circuits, it makes it very difficult to compare to other studies including ours. A recent study showing dopamine is released in ventral striatum in nicotine-dependent persons who smoked compared to those who did not, nonetheless, provides evidence of ventral striatal activity (Brody et al, 2004). Possible explanations for differences between previous PET studies and our MR study include differences in the route of nicotine administration, the use of individual $v s$ multisubject group averaging, ROI compared with voxel-wise analysis, and differences in spatial resolution leading to different partial volume averaging effects.

Previous studies also reported an increase in thalamic blood flow and metabolism following nicotine (Zubieta et al, 2005; Domino et al, 2000b, c), a finding that we did not replicate. It is notable that Rose et al (2003b) demonstrated a complex, inverted-U-shaped, dose-dependent, hemisphere-dependent, response to nicotine in the reticular factor to which the thalamus contributed. Thus, in this welldesigned, constrained study, the regional blood flow response to nicotine were demonstrated to be rather complex, dependent on dose, hemisphere, and nonlinear.

The strengths of this study are the within-subject design and use of a novel, relatively non-invasive method to measure CBF using DSC imaging. The ventral striatum which is considered the center of the reward pathway consists of a medial shell and central core with limited afferent input to these subregions. The borders are difficult to delineate, even at a histochemical level (Haber and McFarland, 1999). Given its small volume and anatomic uncertainties, we believe that an ROI approach to ventral striatum, as used in this study, affords the greatest accuracy. A disadvantage of using ROIs is that it is possible to miss changes in other brain regions. If one were primarily interested in larger cortical areas, then whole brain whole brain approaches such as volumetric brain morphometry (VBM) could be considered. We deliberately chose to maximize spatial resolution of our CBF maps on the targeted region, which necessitated sacrificing full-head coverage. Given the incomplete brain coverage and required spatial smoothing, VBM would not be an option in this study. Limitations of this study are the small sample size and lack of placebo control for nicotine. We had adequate power to detect approximately $10 \%$ difference in CBF. Although our primary intent was to study withdrawal from nicotine, the finding of increased ventral striatum CBF after nicotine gum will have to be confirmed with future placebocontrolled studies of larger sample size. Another limitation is the lack of nicotine blood levels. For each subject, abstinence and the withdrawal syndrome was confirmed with self-report, reduction in exhaled $\mathrm{CO}$, and withdrawal measurements. While less nicotine is absorbed by chewing gum as compared to smoking, significant differences in heart rate, blood pressure, and alleviation of withdrawal symptoms after nicotine gum suggest that levels were sufficient to effect both physiological and subjective changes.

In summary, this is the first paper that specifically investigated whether subjective nicotine-withdrawal symptoms were associated with $\mathrm{CBF}$ changes in neural structures known to be involved positive reinforcement. The time over which withdrawal symptoms emerge, even when as brief as overnight, require absolute measurements which cannot be done using blood-oxygen-level-dependent fMRI techniques. Further, methods that use no radioactivity would be preferable to those involving radioisotope since measurements were repeated four times. Using MR DSC methods, we demonstrated a small, significant effect of nicotine on striatal CBF, but failed to find a main effect of withdrawal. There was correlation between changes in withdrawal and changes in thalamic $\mathrm{CBF}$, however, suggesting that the thalamus may play a more complex if subtle role in the withdrawal state.

\section{ACKNOWLEDGEMENTS}

This work was supported by USPHS grant K08DA1505 to Dr Tanabe. We acknowledge Debra Singel for her assistance in performing the MR perfusion studies and Yungping Wang for her assistance in data analysis.

\section{DISCLOSURE/CONFLICT OF INTEREST}

The authors of this article have no conflicts of interest to disclose.

\section{REFERENCES}

Benowitz NL (1988). Pharmacologic aspect of cigarette smoking and nicotine addiction. N Engl J Med 319: 1318-1339. 
Brody AL, Olmstead RE, London ED, Farahi J, Meyer JH, Grossman P et al (2004). Smoking-induced ventral striatum dopamine release. Am J Psychiatry 161: 1211-1218.

Carroll TJ, Rowley HA, Haughton VM (2003). Automatic calculation of the arterial input function for cerebral perfusion imaging with MR imaging. Radiology 227: 593-600.

CDC (2005). Morbidity and Mortality Weekly Report. Department of Health and Human Services (ed).

Colby SM, Tiffany ST, Shiffman S, Niaura RS (2000). Measuring nicotine dependence among youth: a review of available approaches and instruments. Drug Alcohol Depend 59(Suppl 1): S23-S39.

Cole JWL, Grizzle JE (1966). Applications of multivariate analysis of variance to repeated measruements experiments. Biometrics 22: $810-828$.

Domino EF, Minoshima S, Guthrie S, Ohl L, Ni L, Koeppe RA et al (2000a). Nicotine effects on regional cerebral blood flow in awake, resting tobacco smokers. Synapse 38: 313-321.

Domino EF, Minoshima S, Guthrie S, Ohl L, Ni L, Koeppe RA et al (2000b). Nicotine effects on regional cerebral blood flow in awake, resting tobacco smokers. Synapse 38: 313-321.

Domino EF, Minoshima S, Guthrie SK, Ohl L, Ni L, Koeppe RA et al (2000c). Effects of nicotine on regional cerebral glucose metabolism in awake resting tobacco smokers. Neuroscience 101: 277-282.

Epping-Jordan MP, Watkins SS, Koob GF, Markou A (1998). Dramatic decreases in brain reward function during nicotine withdrawal. Nature 393: 76-79.

Fu SS, Partin MR, Snyder A, An LC, Nelson DB, Clothier B et al (2006). Promoting repeat tobacco dependence treatment: are relapsed smokers interested? Am J Manag Care 12: 235-243.

Ghatan PH, Ingvar M, Eriksson L, Stone-Elander S, Serrander M, Ekberg K et al (1998). Cerebral effects of nicotine during cognition in smokers and non-smokers. Psychopharmacology (Berlin) 136: 179-189.

Gilbert D, McClernon J, Rabinovich N, Sugai C, Plath L, Asgaard G et al (2004). Effects of quitting smoking on EEG activation and attention last for more than 31 days and are more severe with stress, dependence, DRD2 A1 allele, and depressive traits. Nicotine Tob Res 6: 249-267.

Haber SN, McFarland NR (1999). The concept of the ventral striatum in nonhuman primates. Ann NY Acad Sci 877: 33-48.

Harris JG, Kongs S, Allensworth D, Martin L, Tregellas J, Sullivan B et al (2004). Effects of nicotine on cognitive deficits in schizophrenia. Neuropsychopharmacology 29: 1378-1385.

Hildebrand BE, Nomikos GG, Hertel P, Schilstrom B, Svensson TH (1998). Reduced dopamine output in the nucleus accumbens but not in the medial prefrontal cortex in rats displaying a mecamylamine-precipitated nicotine withdrawal syndrome. Brain Res 779: 214-225.

Hughes J, Hatsukami DK (1998). Errors in using tobacco withdrawal scale. Tob Control 7: 92-93.

Hughes JR (2001). Why does smoking so often produce dependence? A somewhat different view. Tob Control 10: 62-64.

Hughes JR, Hatsukami D (1986). Signs and symptoms of tobacco withdrawal. Arch Gen Psychiatry 43: 289-294.

Jarvik M, Killen JD, Varady A, Fortmann SP (1993). The favorite cigarette of the day. J Behav Med 16: 413-422.

Levin JM, Wald LL, Kaufman MJ, Ross MH, Maas LC, Renshaw PF (1998). T1 effects in sequential dynamic susceptibility contrast experiments. J Magn Reson 130: 292-295.

Magnotta VA, Harris G, Andreasen NC, O'Leary DS, Yuh WT, Heckel D (2002). Structural MR image processing using the BRAINS2 toolbox. Comput Med Imaging Graph 26: 251-264.
Markou A, Kosten TR, Koob GF (1998). Neurobiological similarities in depression and drug dependence: a self- medication hypothesis. Neuropsychopharmacology 18: 135-174.

Mawlawi O, Martinez D, Slifstein M, Broft A, Chatterjee R, Hwang DR et al (2001). Imaging human mesolimbic dopamine transmission with positron emission tomography: I. Accuracy and precision of $\mathrm{D}(2)$ receptor parameter measurements in ventral striatum. J Cereb Blood Flow Metab 21: 1034-1057.

Ostergaard L, Weisskoff RM, Chesler DA, Gyldensted C, Rosen BR (1996). High resolution measurement of cerebral blood flow using intravascular tracer bolus passages. Part I: mathematical approach and statistical analysis. Magn Reson Med 36: 715-725.

Piasecki TM, Fiore MC, Baker TB (1998). Profiles in discouragement: two studies of variability in the time course of smoking withdrawal symptoms. J Abnorm Psychol 107: 238-251.

Rempp KA, Brix G, Wenz F, Becker CR, Guckel F, Lorenz WJ (1994b). Quantification of regional cerebral blood flow and volume with dynamic susceptibility contrast-enhanced MR imaging. Radiology 193: 637-641.

Rempp KA, Brix G, Wenz F, Becker CR, Guckel F, Lorenz WJ (1994a). Quantification of regional cerebral blood flow and volume with dynamic susceptibility contrast-enhanced MR imaging. Radiology 193: 637-641.

Rorden C, Brett M (2000). Stereotaxic display of brain lesions. Behav Neurol 12: 191-200.

Rose JE, Behm FM, Westman EC, Mathew RJ, London ED, Hawk $\mathrm{TC}$ et al (2003b). PET studies of the influences of nicotine on neural systems in cigarette smokers. Am J Psychiatry 160: 323-333.

Rose JE, Behm FM, Westman EC, Mathew RJ, London ED, Hawk TC et al (2003a). PET studies of the influences of nicotine on neural systems in cigarette smokers. Am J Psychiatry 160: 323-333.

Rose JE, Behm FM, Westman EC, Mathew RJ, London ED, Hawk TC et al (2003c). PET studies of the influences of nicotine on neural systems in cigarette smokers. Am J Psychiatry 160: 323-333.

Rosen BR, Belliveau J, Buchbinder B, McKinstry RC, Porkka LM, Kennedy DN et al (1991). Contrast agents and cerebral hemodynamics. Magn Reson Med 19: 285-292.

Schneider NG, Jarvik ME, Forsythe AB (1984). Nicotine $v$ s placebo gum in the alleviation of withdrawal during smoking cessation. Addict Behav 9: 149-156.

Stein EA, Pankiewicz J, Harsch HH, Cho JK, Fuller SA, Hoffmann RG et al (1998). Nicotine-induced limbic cortical activation in the human brain: a functional MRI study. Am J Psychiatry 155: 1009-1015.

Watkins SS, Koob GF, Markou A (2000). Neural mechanisms underlying nicotine addiction: acute positive reinforcement and withdrawal. Nicotine Tob Res 2: 19-37.

West R, Hajek P, Belcher M (1989). Time course of cigarette withdrawal symptoms while using nicotine gum. Psychopharmacology (Berlin) 99: 143-145.

Zubieta J, Lombardi U, Minoshima S, Guthrie S, Ni L, Ohl LE et al (2001). Regional cerebral blood flow effects of nicotine in overnight abstinent smokers. Biol Psychiatry 49: 906-913.

Zubieta JK, Heitzeg MM, Xu Y, Koeppe RA, Ni L, Guthrie S et al (2005). Regional cerebral blood flow responses to smoking in tobacco smokers after overnight abstinence. Am J Psychiatry 162: $567-577$. 